\title{
Erratum to: Balanced Sediment Fluxes in Southern California's Mediterranean-Climate Zone Salt Marshes
}

\author{
Jordan A. Rosencranz ${ }^{1,7}$ • Neil K. Ganju ${ }^{2}$. Richard F. Ambrose ${ }^{1,3}$. \\ Sandra M. Brosnahan ${ }^{2}$ • Patrick J. Dickhudt ${ }^{4}$ - Glenn R. Guntenspergen ${ }^{5}$. \\ Glen M. MacDonald ${ }^{1,6} \cdot$ John Y. Takekawa ${ }^{7,8} \cdot$ Karen M. Thorne $^{7}$
}

Published online: 17 February 2016

(C) Coastal and Estuarine Research Federation 2016

\section{Erratum to: Estuaries and Coasts}

DOI 10.1007/s12237-015-0056-y

In the abstract of the original article, the sentence "... while eastern channel storm imports augmented $9200 \mathrm{~kg}$ imported during dry weather" should be replaced by “...while eastern channel storm imports augmented $18000 \mathrm{~kg}$ imported during dry weather." This error did not affect the interpretation of the results. Also, in the section titled, "Suspended Sediment Concentration and Vertical Accretion on the Salt Marsh
Plain," the sentence "... and $30 \%$ at Seal Beach during dry weather from the tidal creek to near creek stations" should be replaced by " .... and $31 \%$ at Seal Beach during dry weather from the tidal creek to near creek stations" This error did not affect the interpretation of the results.

Lastly, in the original article there were several errors in Tables 2 and 3 that also did not impact the interpretation of the results. Following are the corrected tables.

The online version of the original article can be found at http://dx.doi.org/ 10.1007/s12237-015-0056-y.

Jordan A. Rosencranz

jrosencranz@usgs.gov

1 Institute of the Environment and Sustainability, University of California, Los Angeles, CA 90095, USA

2 US Geological Survey, Woods Hole Coastal and Marine Science Center, Woods Hole, MA 02556, USA

3 Department of Environmental Health Sciences, University of California, Los Angeles, CA 90095-1772, USA

4 US Army Corps of Engineers, Field Research Facility, Kitty Hawk, NC 27949-4472, USA

5 US Geological Survey, Patuxent Wildlife Research Center, Superior, WI 54880, USA

6 Department of Geography, University of California, Los Angeles, CA 90095-1524, USA

7 US Geological Survey, Western Ecological Research Center, San Francisco Bay Estuary Field Station, Vallejo, CA 94592, USA

8 National Audubon Society, Science Division, 220 Montgomery Street, San Francisco, CA 94104, USA 
Table 2 Sediment flux components during multi-day storm event in 2014, compared to non-storm periods and entire study period (total)

\begin{tabular}{|c|c|c|c|c|c|c|}
\hline \multirow{2}{*}{$\begin{array}{l}\text { Parameter } \\
\text { Sediment flux component }\end{array}$} & \multicolumn{3}{|c|}{ Seal Beach1 } & \multicolumn{3}{|c|}{ Seal Beach2 } \\
\hline & Storm & Non-storm & Total & Storm & Non-storm & Total \\
\hline Mean advective $(<u><a><c>)(\mathrm{g} / \mathrm{s})$ & -17 & -11 & -11 & -8.5 & 4.9 & 4.2 \\
\hline Mean dispersive $\left(<u^{\prime}><a><c^{\prime}>\right)(\mathrm{g} / \mathrm{s})$ & 23 & 0.39 & 1.4 & 21 & -8.0 & -6.4 \\
\hline Mean stokes drift $\left(u^{\prime} a^{\prime}<c>\right)(\mathrm{g} / \mathrm{s})$ & 26 & 9.3 & 10 & 11 & 8.4 & 8.5 \\
\hline Mean total flux $(\mathrm{g} / \mathrm{s})$ & 39 & -1.3 & 0.50 & 28 & 3.5 & 4.9 \\
\hline Mean total flux normalized by channel area $\left(\mathrm{g} / \mathrm{m}^{2} / \mathrm{s}\right)$ & 0.71 & -0.024 & 0.0091 & 0.29 & 0.037 & 0.052 \\
\hline Advective $(<u><a><c>)(\mathrm{kg})$ & -5400 & $-73,000$ & $-78,000$ & -2600 & 25,000 & 23,000 \\
\hline Dispersive $\left(<u^{\prime}><a><c^{\prime}>\right)(\mathrm{kg})$ & 7200 & 2600 & 9800 & 6500 & $-41,000$ & $-35,000$ \\
\hline Stokes drift $\left(u^{\prime} a^{\prime}<c>\right)(\mathrm{kg})$ & 7900 & 61,000 & 69,000 & 3300 & 43,000 & 46,000 \\
\hline Total flux (kg) & 12,000 & -8700 & 3400 & 8800 & 18,000 & 27,000 \\
\hline
\end{tabular}

Table 3 Average suspended sediment concentration values on marsh surface and in tidal creeks measured in 2014

\begin{tabular}{llll}
\hline Metric & Site & \\
\cline { 2 - 4 } Distance from tidal creek edge & Mugu (storm) & Mugu (no storm) & Seal Beach (no storm) \\
\hline Tidal creek & $264 \pm 61 \mathrm{mg} / \mathrm{L}(\mathrm{n}=12)$ & $51 \pm 5 \mathrm{mg} / \mathrm{L}(\mathrm{n}=12)$ & $58 \pm 7 \mathrm{mg} / \mathrm{L}(\mathrm{n}=27)$ \\
Near $(1-3 \mathrm{~m})$ & $202 \pm 27 \mathrm{mg} / \mathrm{L}(\mathrm{n}=33)$ & $35 \pm 3 \mathrm{mg} / \mathrm{L}(\mathrm{n}=36)$ & $40 \pm 2 \mathrm{mg} / \mathrm{L}(\mathrm{n}=69)$ \\
Mid $(5-10 \mathrm{~m})$ & $214 \pm 30 \mathrm{mg} / \mathrm{L}(\mathrm{n}=33)$ & $38 \pm 4 \mathrm{mg} / \mathrm{L}(\mathrm{n}=36)$ & $39 \pm 1 \mathrm{mg} / \mathrm{L} \mathrm{(n=72)}$ \\
Far $(15-31 \mathrm{~m})$ & $77 \pm 9 \mathrm{mg} / \mathrm{L}(\mathrm{n}=31)$ & $32 \pm 3 \mathrm{mg} / \mathrm{L}(\mathrm{n}=36)$ & $35 \pm 1 \mathrm{mg} / \mathrm{L}(\mathrm{n}=79)$ \\
\hline
\end{tabular}

Error bars indicate a range of \pm 1 standard error 